\title{
Can ab initio simulation really predict properties of materials prior to experimental
}

\author{
YOSHIYUKI KAWAZOE \\ Institute for Materials Research, Tohoku University, Sendai 980-8577, Japan
}

\begin{abstract}
In this paper we have presented all-electron full-potential ab initio simulation method with introduction of mixed-basis, and have cited several typical examples which indicate that it is possible to predict properties of materials prior to experimental. Based on the $a b$ initio calculation of the total energy, cluster variation, and direct methods function, it is possible to bridge the limited scheme of the ab initio treatment to real complex materials. Furthermore, to overcome the limited computer power, we have developed parallel processing codes and tested their efficiencies as well.
\end{abstract}

Keywords. Materials design; $a b$ initio; cluster variation method; direct method; parallel processing.

\section{Introduction}

In recent years, designing of materials based only on experience has come up against a stone wall, since, according to industrial requirement for materials having higher and higher physical and chemical properties, the materials to be developed have become increasingly complex and/or are required to be controlled atomistically. With explosion in the number of combination of elements that require increasing expenditure and time for experimental studies, a need for introduction of a new method of computer simulation to predict structures of materials and their properties in computer prior to real experiment, has arisen.

By using the largest supercomputing system available, it is possible to calculate band structure of a system containing several hundreds of atoms in a unit cell. This size is good enough to discuss industrially useful materials, and it is possible to predict physical and chemical properties of materials in detail. To relax the system completely with the first-principles local density approximation (LDA) calculations, the present-day supercomputer power is however not quite sufficient, since it is only limited up to 10 atoms in a unit cell-normally, we assume some symmetries and try to relax around these symmetries! Furthermore, the first-principles molecular dynamics is also limited to several picoseconds in time duration, although some chemical reactions do take place within this time period and it is possible to simulate dynamic behaviour of these reactions. Most of the interesting chemical and physical processes however take longer time and therefore it is not possible to trace them.

In the following sections, all-electron full-potential $a b$ initio simulation method with mixed-basis is introduced to show the possibility to predict properties of materials by the present-day state-of-art theoretical method. However, since by using only the first-principles calculations, the size and the time duration to be treated are limited, therefore cluster variation method (CVM) and direct method, which enable more realistic handling of systems, are indicated. Finally, to overcome the present limitations of simulation based on the standard approximations, concepts of beyond the present theories are discussed.

\section{All-electron full-potential $a b$ initio simulation method with mixed-basis}

In this section, the computational scheme in an allelectron full-potential mixed-basis approach is described briefly. The mixed-basis approach was originally introduced in the $a b$ initio calculation of transition metal elements by Louie et al (1979) in order to treat efficiently the spatial locality and asymmetry of $3 d$ orbitals with limited number of basis functions. It is a technique to expand the wavefunctions in terms of both plane waves (PW) and atomic orbitals (AO). They applied this approach in pseudopotential formalism. The pseudopotential scheme allows one to replace a strong electronion Coulomb potential with a soft potential.

However, it has often been pointed out that the description of wave functions using only PWs is not easy, in particular for $2 p$ orbitals in the second-row elements and $3 d$ orbitals in transition metal elements, even if an efficient pseudopotential is used. In our all-electron fullpotential, mixed-basis approach, both PWs and AOs are used to expand the true wave functions including the core.

The all-electron, mixed-basis approach for band structure calculations has been successfully applied to the experimental results of aligned $\mathrm{C}_{60}$ on $\mathrm{Cu}(111)$ (figure 1) (Maruyama et al 1995), and to fcc and sc $\mathrm{C}_{60}$ (see figure 2) (Gu et al 1994a, b; Ohno et al 1996a). It has been applied to dynamics as well (figure 3) (Ohno et al 
1996b, c; Ohtsuki et al 1998). In the mixed-basis formalism, the basis is composed both of PW and AO. Since the AOs are not orthogonal to the PWs, it is necessary to orthogonalize the basis at each iteration step. The exchange-correlation potential is evaluated in real space under the local density approximation. In the present formalism, the charge density and the self-consistent potential are evaluated either in real space (for AO-related part) or in Fourier space (for PW-related part).

Once the potential is determined, the Hamiltonian matrix elements have to be evaluated. In the potential matrix elements, there are three types of possible combinations: PW-PW, PW-AO, and AO-AO. As in the PW approach, it is not necessary to register the PW-PW matrix elements in computer-memory, since the PW-PW block does not change once the diagonal transformation has occurred. The other combinations, PW-AO and AO$\mathrm{AO}$, are conveniently calculated in the real space inside each atomic sphere.

\section{More than the standard ab initio calculation}

To carry out the numerical calculations of above samples by supercomputer, it typically takes several $10 \mathrm{~h}$ of CPU time with order of $1 \mathrm{~GB}$ of main memory. This size is the limitation under standard condition of supercomputer time sharing. To study more complex systems, using quantum theory, with larger number of atoms, several attempts have been made for example, tight-binding modelling, wherein the potential normally functions nicely around the region where the potential is determined. In this chapter we have however introduced more sophisticated methods
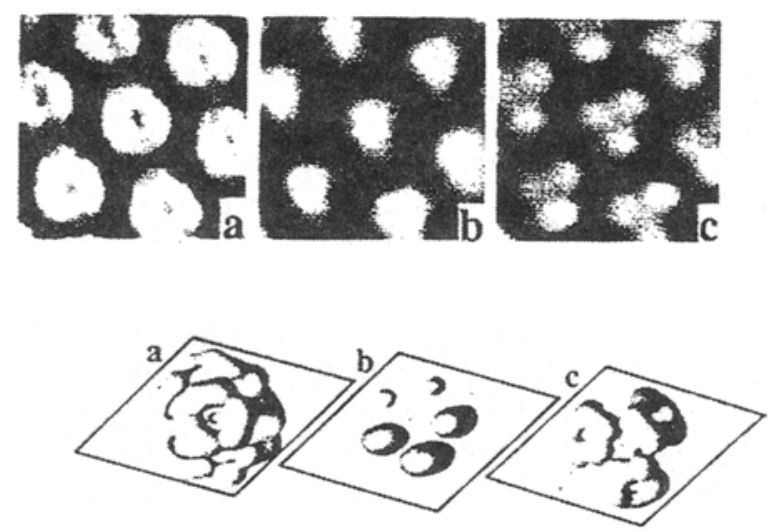

Figure 1. Upper three figures show STM images of $\mathrm{C}_{60}$ selforganized on $\mathrm{Cu}(1.11)$ surface around the gap. a, b, and $\mathrm{c}$ indicated different bias voltages corresponding to different excitations. Lower three figures are the results of the mixedbasis $a b$ initio calculation, which can explain the reason of the observed patterns in the above figures (symmetries of charge distribution is determined by the electronic state). to apply to more complex systems by using the results of $a b$ initio calculations.

\subsection{Cluster variation method}

The standard $a b$ initio LDA calculation gives us information on the ground state total energy and stable structure of the materials together with their physicochemical properties. However, it is necessary to study thermodynamic properties to reveal dynamic behaviours of materials at finite temperatures. To achieve this aim, cluster variation method (CVM) has been used to determine the phase diagram by estimating the free energy, using the energy parameters for clusters in complex systems.

Moreover, CVM can also be applied to determine site preference of ternary additions in alloys. Thus, we have studied the site preferences of transition metals in $\mathrm{Ni}_{3} \mathrm{Al}$ and other important alloy systems (Sluiter and Kawazoe 1995).

Another application of CVM is in the study of the dynamic behaviour of the interface. For example, the $\mathrm{Al}-\mathrm{Al}_{3} \mathrm{Li}$ system has been studied (Sluiter et al 1996). We have used a supercell consisting of $38 \mathrm{fcc}$ cubes in the calculation, including (100) interface boundary (IPB). A part of the derived results is shown in figure 4 . It clearly

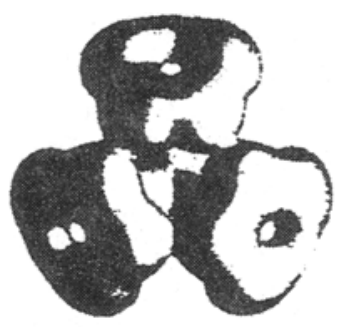

(a)

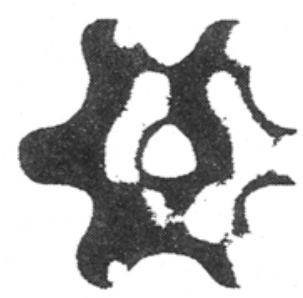

(b)

Figure 2. Isosurface of partial charge density (PCD) distribution. (a) represents PCD composed of the highest 12 occupied molecular orbital (HOMO, $h_{u}$ ) bands, while (b) PCD of the lowest 12 unoccupied molecular orbital (LUMO, $t_{14}$ ) bands. 
shows that at the IPB, the $\mathrm{Li}$ concentration varies from one phase to another: at $400 \mathrm{~K}$ the thickness of this variation is about 4 lattice constants, and it is a linear function of temperature.

\subsection{Direct method}

Another method to treat problems in materials science, based on the $a b$ initio calculations, is the direct method, described by Parlinski et al (1997). This has been recently used to study phonon dispersions and phase transitions in crystalline solids.

The calculation of phonon frequencies of the crystal is one of the fundamental subjects to study the phase stability, phase transition, and thermodynamics of the crystal. By using another $a b$ initio calculation, the linear response method, it is difficult to determine phonon dispersions, since the dielectric matrix must be calculated in terms of the electronic eigenfunctions of a perfect crystal (King-Smith and Needs 1990).

In the direct method (Parlinski et al 1997), however, the dynamical matrix is constructed using cumulant force

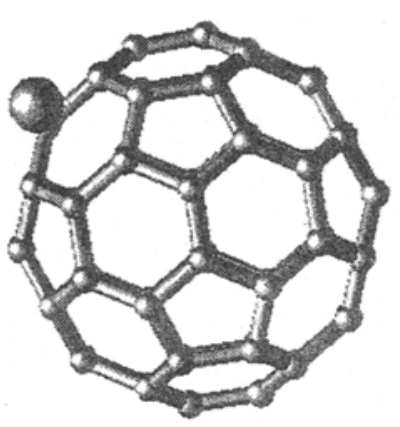

$0.0 \mathrm{fs}$

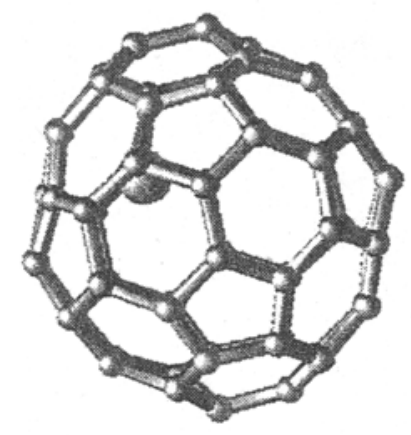

$40.0 \mathrm{fs}$

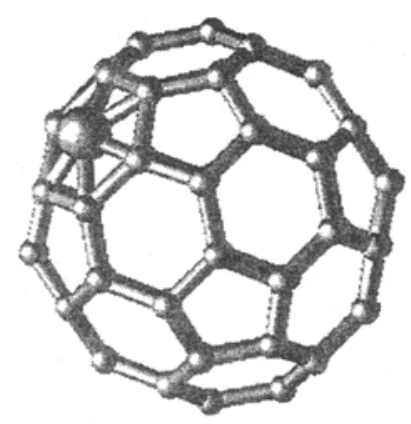

$20.0 \mathrm{fs}$

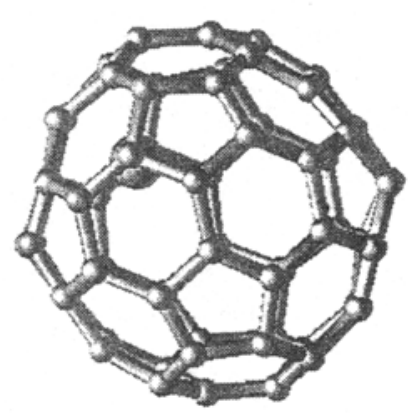

$100.0 \mathrm{fs}$
Figure 3. Snapshots of the $\mathrm{Li}$ insertion process to $\mathrm{C}_{60}$. At the initial kinetic energy of 5 to $20 \mathrm{eV}, \mathrm{Li}$ atom can penetrate into $\mathrm{C}_{60}$ through hexagon of carbon atoms on the $\mathrm{C}_{60}$ surface and trapped closed the cage. This metallic atom included fullerene is expected to be a stable high- $T_{\mathrm{c}}$ material. constants, which are determined numerically by HellmanFynman force, and are subsequently, solved to obtain phonon dispersions.

We have successfully applied the direct method to study the structural phase transition in cubic $\mathrm{ZrO}_{2}$. The force constants were determined from the HellmanFynman forces induced by displacement of atoms in a $2 \times 2 \times 2$ fcc supercell. The calculated phonon dispersions, as shown in figure 5 , clearly show a soft mode at $\mathrm{X}$ point, which corresponds to the experimentally confirmed cubic to tetragonal structural phase transition.

\section{Beyond the present approximations}

Most of the text books on solid state physics start with the statement that the adiabatic Born-Oppenheimer, or BO, approximation is good enough, since nucleus is almost
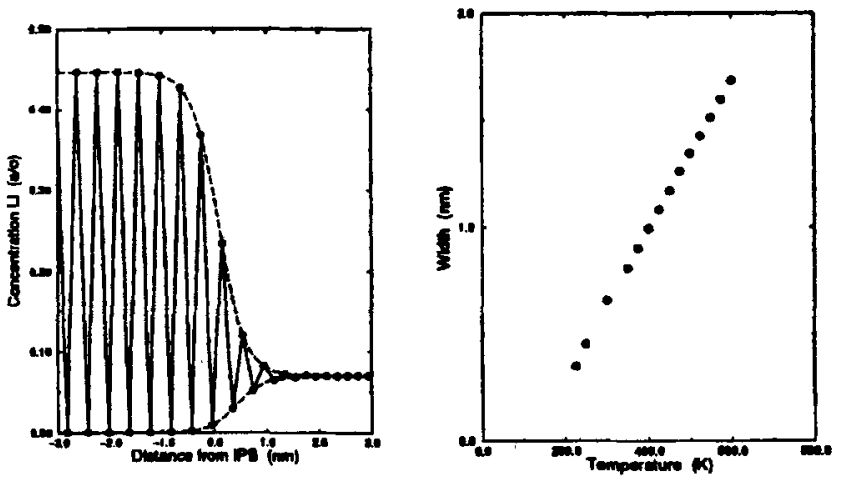

Figure 4. Li concentration averaged over (100) planes parallel to the IPB as a function of distance (in units of the lattice constant) at $400 \mathrm{~K}$. The solid and dotted lines are drawn for the eye.

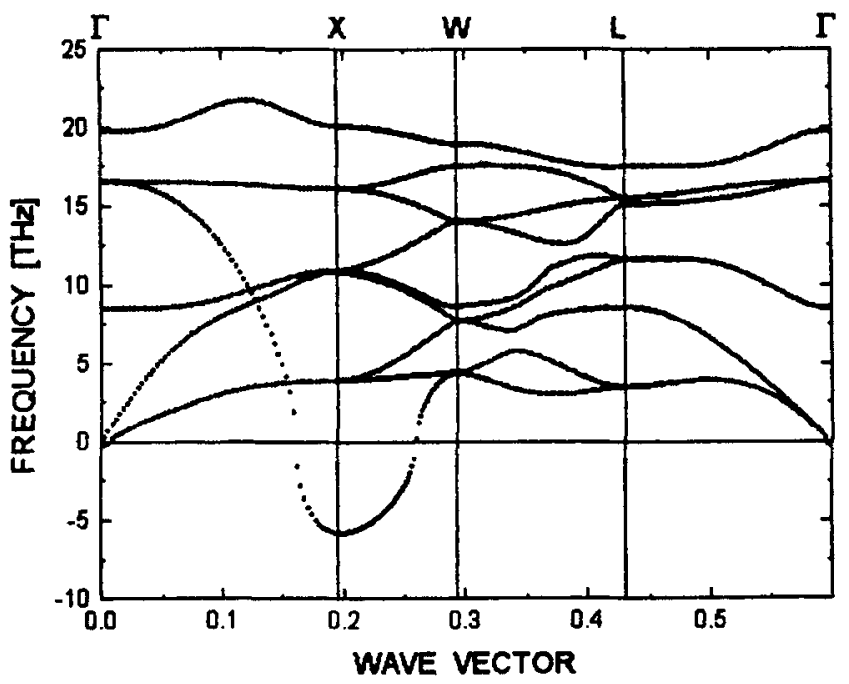

Figure 5. Calculated phonon dispersions of $\mathrm{ZrO}_{2}$ in the cubic structure. At the $X$ point it clearly indicates the existence of soft mode, which corresponds to a structural phase transition observed experimentally in this material. 
2,000 times heavier than the electron. However, in some cases, it is not so simple. For example, to simulate chemical reactions, we should consider that the reactions depend strongly on temperature, and that many branches occur with variety of branching ratios. This means that the reaction is not taking place only on the $B O$ surface (beyond $\mathrm{BO}$ ). The above-mentioned insertion process of $\mathrm{Li}$ into $\mathrm{C}_{60}$ is one such case, since $\mathrm{Li}$ passes the $\mathrm{C}_{60}$ very rapidly. Recently, supercritical water (water under high pressure and at high temperature) has attracted attention, since it is very reactive to be able to resolve even PET bottles. This is very important for recycling of materials. This special phase of water has been studied extensively, both experimentally as well as theoretically. The understanding of the supercritical water is not yet completed, especially, since theoretical treatment has been limited to the classical molecular dynamics. To study the fundamental behaviour of this extraordinarily reactive water, 'beyond BO' theory is important, for under such condition protons vibrate very strongly and the electron clouds are separated from the nucleus.

To overcome the present level of BO approximation, we have tested three Fermion systems, $\mathrm{H}^{-}$and $\mathrm{H}_{2}^{+}$, almost analytically and have shown that the quantization of kinetic energies of proton is essential to discuss these quantum systems (Farajian et al, to be published).

Since present-day standard local density approximation (LDA) and/or generalized gradient approximation (GGA) is known to be accurate only for the ground state without degeneracy, we should consider including excited states as well in order to obtain really meaningful simulation results which could be compared with most of the experimentals, such as band gap in semiconductor. To achieve this aim, we are considering including GW approximation (quasi particle treatment) into the first principles band calculation, and into the molecular dynamics code.

The last, 'go beyond', story is to solve the timedependent Schröedinger equation-as it is. This approach can be named beyond Car-Parrinnello (CP), since it does not use any fictitious mass in its formulation. We have tested this approach for the $\mathrm{Li}$ inclusion procedure into $\mathrm{C}_{60}$, since for this case calculation of the variational force is simply as a derivative of time.

\section{Conclusions}

An LDA code with all-electron full-potential mixed-basis formulation is introduced and typical examples of the results, by using the program, indicate that it is really one of the best existing ab initio codes within LDA and GGA, and that it can treat molecular dynamics far better than FLAPW.
However, the use of ab initio calculation is still limited only to treating real materials having interesting properties, since even the largest computer systems handles only up to several hundreds of atoms in a unit cell. To fill this gap, cluster variation method and direct method have been applied to simulate dynamical behaviours of complex materials.

It is true that the first-principles calculation has no phenomenological parameters, but it uses a lot of approximations, such as LDA. We are trying to overcome this drawback by expanding the standard approximation

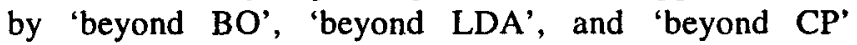
approaches. We expect these new levels of theories to simulate more realistically the physical and chemical behaviours of materials.

\section{Acknowledgements}

The author is grateful to his collaborators. Prof. K Ohno and $\operatorname{Dr} \mathrm{Y}$ Maruyama have done the main part of the formulation and program development of mixed-basis code. Prof. B -L Gu contributed much in the first stage of the program development. Profs $K$ Parlinski and Q Sun, Drs K Esfarjani, M Sluiter, Z -Q Li, L Zhou, Y Hashi and $\mathrm{J}-\mathrm{Z} \mathrm{Yu}$ and Mrs Shiga, Ishii and Farajian worked in various applications of the code including CVM and direct method. The supercomputing system HITAC S$3800 / 380$ was maintained by the crew of the Computer Science Group at IMR, Tohoku University.

\section{References}

Gu B -L, Maruyama Y, Yu J -Z, Ohno K and Kawazoe Y 1994a Phys. Rev. B49 16202

Gu B -L, Maruyama Y, Yu J -Z, Ohno K and Kawazoe Y 1994b MRS Symposium Proceedings vol. 349 p. 289

King-Smith R D and Needs R J $1990 \mathrm{~J}$. Phys. Condensed Matter 23431

Louie S G, Ho K M and Cohen M L 1979 Phys. Rev. B19 1774

Maruyama Y, Ohno K and Kawazoe Y 1995 Phys. Rev. 152 2070

Ohno K, Maruyama Y, Takahashi M, Yu J -Z, Gu B -L and Kawazoe Y 1996a Surf. Rev. Lett. 3735

Ohno K, Maruyama Y and Kawazoe Y 1996b Phys. Rev. B53 4078

Ohno K, Maruyama Y, Esfarjani K, Kawazoe Y, Sato N, Hatakeyama R, Hirata T and Niwano M 1996 Phys. Rev. Lett. 763590

Ohtsuki T, Ohno K, Shiga K, Kawazoe $Y$, Maruyama $Y$ and Masumoto K 1998 Phys. Rev. Lett. 81967

Parlinski K, Li Z -Q and Kawazoe Y 1997 Phys. Rev. Lett. 78 4063

Sluiter M and Kawazoe Y 1995 Phys. Rev. B51 4062

Sluiter M, Asta M and Kawazoe Y 1996 Sci. Rep. RITU A41 97 